\title{
The Role of Space Dimension on the Blow up for a Reaction-Diffusion Equation
}

\author{
Zhilei Liang \\ School of Economic Mathematics, Southwestern University of Finance and Economics, Chengdu, China \\ E-mail: zhilei0592@gmail.com
}

Received March 9, 2011; revised March 22, 2011; accepted March 27, 2011

\section{Abstract}

This paper deals with the doubly degenerate reaction-diffusion equation $\frac{\partial u}{\partial t}=\operatorname{div}\left(u^{m-1}|\nabla u|^{\lambda-1} \nabla u\right)+u^{q}$, $x \in B(0,1), \quad t>0$, where $m \geq 1, \quad \lambda \geq 1, q \geq m+\lambda-1>1$ and $B(0,1)$ denotes a unit ball in $R^{N}$ with the center in origin. We prove that the blow up phenomenon can be restrained if the space dimension $N$ is taken sufficiently large. Moreover, the critical condition guaranteeing the absence (or occurrence) of the blow up is achieved.

Keywords: Reaction-Diffusion, Source Term, Space Dimension, Blow up

\section{Introduction}

The blow up phenomenon in parabolic equation has been object of active research in recent years [1-4]. It has attracted lots of interests and attentions because of its physical importance and mathematical challenge. Here we formulate the condition that guarantees the absence of the blow up in terms of space dimension. In order to fix ideas, we consider the following problem

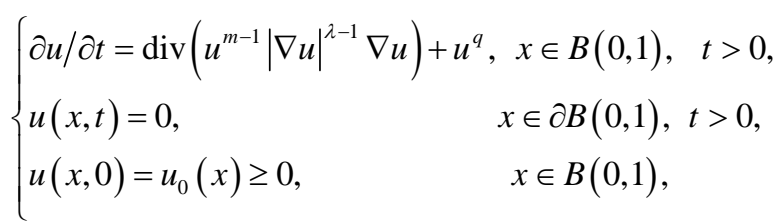

where $m \geq 1, \lambda \geq 1, q \geq m+\lambda-1>1$, and $B(0,1)$ is a unit ball in $R^{N}$ with the center in origin. The initial function $u_{0}(x)$ is assumed to be positive bounded. Problem (1.1) is the well known non-Newtonian polytropic filtration equation, which comes up in a wide variety of physical contexts. The local in time existence and the comparison principle of nonnegative weak solutions have been established in [5], see also [6]. Due to the possible degeneration at the level $u=0$ or $|\nabla u|=0$, we understand the weak solution in distributional sense as follows

Definition 1.1 Let $\sigma=(m+\lambda-1) / \lambda$. A nonnegative $u \in L^{\infty}\left(B(0,1) \times(0, t) \cap C(0, t) ; L^{2}(B(0,1))\right)$ with $\left|\nabla u^{\sigma}\right|^{\lambda+1} \in L^{1}(B(0,1) \times(0, t))$ is said to be a weak solution of (1.1), if the integral identity

$$
\begin{aligned}
& \int_{0}^{t} \int_{B}\left\{u \phi_{t}-u^{m-1}|\nabla u|^{\lambda-1} \nabla u \nabla \phi+u^{q} \phi\right\} \mathrm{d} x \mathrm{~d} s \\
& +\int_{B} u_{0}(x) \phi(x, 0) \mathrm{d} x=0
\end{aligned}
$$

is true for all test function $\phi \in C_{0}^{\infty}(B(0,1) \times[0, T))$.

When it comes to blow up, we mean that the solution of (1.1) exists for $t \in[0, T)$ and become unbounded as $t \rightarrow T$ for some $T<\infty$.

In this paper, we study the influencing factor of space dimension $N$ on the blow up phenomenon for the Dirichlet problem (1.1). Such question was first investigated by A. Tersenov for a heat equation with a nonlinear source. By analyzing the stationary equation and then using the comparison theorem, the author proved in [3] that if the space dimension $N$ is chosen large enough, the solutions exist for all positive time. In other word, the high dimension plays a preventive role on the occurrence of the blow up. In this current paper we extend the results obtained in [3] to a doubly degenerate equation. The below theorem is our first result.

Theorem 1.1 Given an initial function $u_{0}(x)$ and $a$ constant $M>0$, there is a $N^{*}=N^{*}\left(u_{0}(x), M\right)$, such that for every space dimension $N>N^{*}$, the solution $u\left(x, t ; u_{0}\right)$ of (1.1) satisfies

$$
u\left(x, t ; u_{0}\right) \leq\left\|u_{0}\right\|_{\infty}+M, \forall t \geq 0 .
$$

In particular, 


$$
\begin{aligned}
N^{*} & =N^{*}\left(u_{0}, M\right) \\
& =\left(\frac{\lambda+m-1}{\lambda+1}\right)^{\lambda} \cdot\left(\frac{\left(\left\|u_{0}\right\|_{\infty}+M\right)^{q / \lambda}}{\left(\left\|u_{0}\right\|_{\infty}+M\right)^{\sigma}-\left(\left\|u_{0}\right\|_{\infty}\right)^{\sigma}}\right)^{\lambda},
\end{aligned}
$$

where $\sigma=(m+\lambda-1) / \lambda$. Moreover, $N^{*} \rightarrow \infty$ as $\left\|u_{0}\right\|_{\infty} \rightarrow \infty$.

Just as described in Theorem 1.1, there exists a critical $N^{*}$ which depends on the given $u_{0}(x)$, such that the blow up can be avoided if the space dimension $N$ satisfies $N>N^{*}$. On the other hand, for a given space dimension $N$, there also exists a critical initial datum $u_{0}^{*}(x)$ such that all the non-trivial solutions occur blow up so long as the size of their initial datum is larger than that of $u_{0}^{*}(x)$. To demonstrate this we first present the following proposition

Proposition 1.1 For a given space dimension $N$, there exist positive constants $A$, $a$, which depend on $N$, as well as $m, \lambda, q$, such that the function

$$
u_{-}(x, t ; N)=(T-t)^{-1 /(q-1)} \vartheta(\xi),
$$

with $\quad \vartheta(\xi)=A\left[1-(\xi / a)^{(\lambda+1) / \lambda}\right]_{+}^{\lambda /(\sigma \lambda-1)}$, $\xi=|x| /(T-t)^{\alpha}$ and $\alpha=(q-\sigma \lambda) /((\lambda+1)(q-1)) \geq 0$, solves the inequality

$$
\frac{\partial u}{\partial t} \leq \operatorname{div}\left(u^{m-1}|\nabla u|^{\lambda-1} \nabla u\right)+u^{q}, x \in R^{N}, t \in(0, T) .
$$

The proof of Proposition 1.1 is available in the final Section 3.

Because of $\alpha=(q-\sigma \lambda) /((\lambda+1)(q-1)) \geq 0$, the support of $u_{-}(\cdot, t ; N)$ falls into the set $\left\{x:|x| \leq a(T-t)^{\alpha} \leq a T^{\alpha}\right\}$. So, for a fixed $N$, we choose $T$ so small such that $a T^{\alpha} \leq 1$, and therefore,

$$
\operatorname{supp} u_{-}(\cdot, 0 ; N) \subset B(0,1) \text {. }
$$

We are ready to state our second result

Theorem 1.2 Suppose that (1.5) holds for a given space dimension $N$, then there exists a critical initial datum $u_{0}^{*}(x)=u_{-}(x, 0 ; N)$, such that for all initial data $u_{0}$ satisfying

$$
u_{0}(x) \geq u_{0}^{*}(x)=u_{-}(x, 0 ; N),
$$

the solution $u\left(x, t ; u_{0}\right)$ occur blow up before T. Moreover, inequality (1.6), along with (3.7) (see Section 3 below), leads to

$$
\left\|u_{0}^{*}\right\|_{\infty}=\left\|u_{-}(\cdot, 0 ; N)\right\|_{\infty}=A \rightarrow+\infty,(N \rightarrow \infty) .
$$

Remark 1.1 Theorems 1.1 and 1.2 are also valid for a general convex domain.

\section{Proof of the Theorem 1.1}

This section is devoted to the proof of Theorem 1.1. First of all, let us analyze the radially symmetric solution $U(r)$ of the stationary equation to (1.1), i.e.,

$$
\frac{1}{r^{N-1}}\left(r^{N-1} U^{m-1}\left|U^{\prime}\right|^{\lambda-1} U^{\prime}\right)^{\prime}+U^{q}=0, \quad r=|x| \geq 0,
$$

with initial conditions

$$
U(0)=K>0, U^{\prime}(0)=0 .
$$

The local and unique positive solution of (2.1)-(2.2) follows from the analysis of the equivalent integral equation, by using Banach contraction mapping theorem. Moreover, the solution is decreasing and can be extended whenever it is positive. (See [2] and [7].) Let $r^{*}=\sup \{r: U(r)>0, r>0\}$. Integrating (2.1) over $(0, r), r \in\left(0, r^{*}\right)$, yields

$$
r^{N-1} U^{m-1}\left|U^{\prime}\right|^{\lambda-1} U^{\prime}=-\int_{0}^{r} U^{q} s^{N-1} \mathrm{~d} s,
$$

which implies

$$
r^{N-1} U^{m-1}\left(-U^{\prime}\right)^{\lambda}=\int_{0}^{r} U^{q} s^{N-1} \mathrm{~d} s \leq K^{q} \frac{1}{N} r^{N} .
$$

i.e.,

$$
\left(U^{\sigma}\right)^{\prime}(r) \geq-\sigma\left(K^{q} \frac{r}{N}\right)^{\frac{1}{\lambda}}
$$

Integrating (2.4) once more to get

$$
\begin{aligned}
\left(U^{\sigma}\right)(r) & \geq K^{\sigma}-\sigma\left(\frac{K^{q}}{N}\right)^{\frac{1}{\lambda}} \int_{0}^{r} s^{\frac{1}{\lambda}} \mathrm{d} s \\
& =K^{\sigma}-\frac{\lambda+m-1}{\lambda+1}\left(\frac{1}{N}\right)^{\frac{1}{\lambda}} K^{\frac{q}{\lambda}} r^{\frac{\lambda+1}{\lambda}},
\end{aligned}
$$

i.e.,

$$
U(r) \geq\left[K^{\sigma}-\frac{\lambda+m-1}{\lambda+1}\left(\frac{1}{N}\right)^{\frac{1}{\lambda}} K^{\frac{q}{\lambda}} r^{\frac{\lambda+1}{\lambda}}\right]^{\frac{1}{\sigma}}, 0 \leq r \leq r^{*} .
$$

Clearly,

$$
r^{*} \geq\left(\frac{\lambda+1}{\lambda+m-1}\right)^{\frac{\lambda}{\lambda+1}} N^{\frac{1}{\lambda+1}} K^{\frac{\lambda+m-1-q}{\lambda+1}} .
$$

Since the initial function $u_{0}(x) \in L^{\infty}(B(0,1))$, from (2.5) we have

$$
u_{0}(x) \leq U(|x|), \forall x \in B(0,1),
$$

provided

$$
U(1) \geq\left[K^{\sigma}-\frac{\lambda+m-1}{\lambda+1}\left(\frac{1}{N}\right)^{\frac{1}{\lambda}} K^{\frac{q}{\lambda}}\right]^{\frac{1}{\sigma}} \geq\left\|u_{0}\right\|_{\infty},
$$


which is reduced to

$$
N \geq\left[\frac{\lambda+m-1}{\lambda+1} \cdot \frac{K^{\frac{q}{\lambda}}}{K^{\sigma}-\left(\left\|u_{0}\right\|_{\infty}\right)^{\sigma}}\right]^{\lambda}=N^{*} .
$$

To guarantee the validity of (2.7), it suffices to take $K=\left\|u_{0}\right\|_{\infty}+M$ with a constant $M>0$. That is,

$$
N \geq\left[\frac{\lambda+m-1}{\lambda+1} \cdot \frac{\left(\left\|u_{0}\right\|_{\infty}+M\right)^{\frac{q}{\lambda}}}{\left(\left\|u_{0}\right\|_{\infty}+M\right)^{\sigma}-\left(\left\|u_{0}\right\|_{\infty}\right)^{\sigma}}\right]^{\lambda}=N^{*} .
$$

Apparently, (2.7) also ensures

$$
U(|x|) \geq 0=u(x, t) \text {, for all } x \in \partial B(0,1), t>0 .
$$

In terms of (2.7) and (2.8), the comparison theorem conclude

$$
u(x, t) \leq U(x), \forall x \in B(0, R), t>0
$$

This deduces that $u(x, t) \leq U(x) \leq U(0)=\left\|u_{0}\right\|_{\infty}+M, \forall x \in B(0,1), t>0$.

This completes the proof of Theorem 1.1.

Remark 2.1 In case of $q<\lambda+m-1$, all the solutions of the (1.1) can be bounded by $U(r)$ for arbitrary $N$, only if the $U(0)=K$ is chosen large enough (see (2.5)). We also refer to [4].

\section{Proof of Theorem 1.2}

The proof of Theorem 1.2 is a direct consequence of Proposition 1.1. So the remain task is to prove the validity of the Proposition 1.1. In fact, to verify the function $u_{-}(x, t ; N)=(T-t)^{-\frac{1}{q-1}} \vartheta(\xi)$ satisfies (1.4), we turn to prove the following equivalent inequality obtained after relatively computation

$$
\begin{aligned}
& \frac{1}{\xi^{N-1}}\left(\xi^{N-1} \vartheta^{m-1}\left|\vartheta^{\prime}\right|^{\lambda-1} \vartheta^{\prime}\right)^{\prime}-\alpha \xi \vartheta^{\prime} \\
& -\frac{1}{q-1} \vartheta+\vartheta^{q} \geq 0, \quad \xi \neq a,
\end{aligned}
$$

in which ' $=\mathrm{d} /(\mathrm{d} \xi)$. Due to the explicit form $\vartheta(\xi)=\left[1-(\xi / a)^{(\lambda+1) / \lambda}\right]_{+}$, the derivative of $\vartheta$ is nonincreasing, i.e.,

$$
\vartheta^{\prime}=-\frac{A}{a} \frac{\lambda+1}{\sigma \lambda-1}\left[1-\left(\frac{\xi}{a}\right)^{\frac{\lambda+1}{\lambda}}\right]_{+}^{\frac{\lambda}{\sigma \lambda-1}-1}\left(\frac{\xi}{a}\right)^{\frac{\lambda+1}{\lambda}-1} \leq 0 .
$$

By this we compute

$$
\begin{aligned}
& \frac{1}{\xi^{N-1}}\left(\xi^{N-1} \vartheta^{m-1}\left|\vartheta^{\prime}\right|^{\lambda-1} \vartheta^{\prime}\right)^{\prime} \\
= & \frac{1}{\sigma} \frac{1}{\xi^{N-1}}\left(\xi^{N-1}\left(\left|\left(\vartheta^{\sigma}\right)^{\prime}\right|^{\lambda-1}\left(\vartheta^{\sigma}\right)^{\prime}\right)^{\prime}\right) \\
= & \frac{1}{\sigma}\left[\left(\left|\left(\vartheta^{\sigma}\right)^{\prime}\right|^{\lambda-1}\left(\vartheta^{\sigma}\right)^{\prime}\right)^{\prime}+\frac{N-1}{\xi}\left|\left(\vartheta^{\sigma}\right)^{\prime}\right|^{\lambda-1}\left(\vartheta^{\sigma}\right)^{\prime}\right] \\
= & I_{1}+I_{2} .
\end{aligned}
$$

A direct but tedious computation shows

$$
\begin{aligned}
I_{1}= & \frac{1}{\sigma a}\left(\frac{A \sigma}{a}\right)^{\lambda}\left(\frac{\sigma(\lambda+1)}{\sigma \lambda-1}\right)^{\lambda} \frac{\lambda+1}{\sigma \lambda-1} \varpi^{\frac{\lambda}{\sigma \lambda-1}-1} \\
& -\frac{1}{\sigma a}\left(\frac{A \sigma}{a}\right)^{\lambda}\left(\frac{\sigma(\lambda+1)}{\sigma \lambda-1}\right)^{\lambda}\left(\frac{\lambda+1}{\sigma \lambda-1}+1\right) \varpi^{\frac{\lambda}{\sigma \lambda-1}},
\end{aligned}
$$

and

$$
I_{2}=-(N-1) \frac{1}{\sigma a}\left(\frac{A \sigma}{a}\right)^{\lambda}\left(\frac{\sigma(\lambda+1)}{\sigma \lambda-1}\right)^{\lambda} \varpi^{\frac{\lambda}{\sigma \lambda-1}},
$$

where $\varpi=\left[1-\left(\frac{\xi}{a}\right)^{\frac{\lambda+1}{\lambda}}\right]_{+}$. Hence, (3.2) is equals to

$$
\begin{aligned}
& \frac{1}{\xi^{N-1}}\left(\xi^{N-1} \vartheta^{m-1}\left|\vartheta^{\prime}\right|^{\lambda-1} \vartheta^{\prime}\right)^{\prime} \\
= & \frac{1}{\sigma a}\left(\frac{A^{\sigma}}{a}\right)^{\lambda}\left(\frac{\sigma(\lambda+1)}{\sigma \lambda-1}\right)^{\lambda} \frac{\lambda+1}{\sigma \lambda-1} \varpi^{\frac{\lambda}{\sigma \lambda-1}-1} \\
& -\frac{1}{\sigma a}\left(\frac{A^{\sigma}}{a}\right)^{\lambda}\left(\frac{\sigma(\lambda+1)}{\sigma \lambda-1}\right)^{\lambda}\left(N+\frac{\lambda+1}{\sigma \lambda-1}\right) \varpi^{\frac{\lambda}{\sigma \lambda-1}} .
\end{aligned}
$$

Similarly,

$$
\begin{aligned}
& -\alpha \xi \vartheta^{\prime}=-\alpha A \frac{\lambda+1}{\sigma \lambda-1} \varpi^{\frac{\lambda}{\sigma \lambda-1}}+\alpha A \frac{\lambda+1}{\sigma \lambda-1} \varpi^{\frac{\lambda}{\sigma \lambda-1}-1}, \\
& -\frac{1}{q-1} \vartheta+\vartheta^{q}=-\frac{1}{q-1} A \varpi^{\frac{\lambda}{\sigma \lambda-1}}+A^{q} \varpi^{\frac{q \lambda}{\sigma \lambda-1}} .
\end{aligned}
$$

Summing up (3.3)-(3.5), we receive

$$
\Phi(\varpi)=k-l \varpi+A^{q-1} \varpi^{\frac{q \lambda-\lambda}{\sigma \lambda-1}+1} \geq 0,
$$

where

$$
k=\frac{1}{\sigma}\left(\frac{A^{\lambda \sigma-1}}{a^{\lambda+1}}\right)\left(\frac{\sigma(\lambda+1)}{\sigma \lambda-1}\right)^{\lambda} \frac{\lambda+1}{\sigma \lambda-1}+\alpha \frac{\lambda+1}{\sigma \lambda-1},
$$

and 


$$
\begin{aligned}
l= & \frac{1}{\sigma}\left(\frac{A^{\lambda \sigma-1}}{a^{\lambda+1}}\right)\left(\frac{\sigma(\lambda+1)}{\sigma \lambda-1}\right)^{\lambda}\left(N+\frac{\lambda+1}{\sigma \lambda-1}\right) \\
& +\alpha \frac{\lambda+1}{\sigma \lambda-1}+\frac{1}{q-1} .
\end{aligned}
$$

inequality (3.6) holds so long as

$$
\begin{aligned}
\Phi(\varpi) & =k-l \varpi+A^{q-1} \varpi^{\frac{q \lambda-\lambda}{\sigma \lambda}-1} \\
& \geq k-l+A^{q-1}\left(\frac{k}{l}\right)^{\frac{q \lambda-\lambda}{\sigma \lambda}-1} \geq 0
\end{aligned}
$$

Firstly, because $\Phi(0)=k>0$, we have $\Phi(\varpi) \geq 0$ for all $\varpi \in[0, k / l]$ with $k / l \in(0,1)$. As to $\varpi \in(k / l, 1]$,

which is valid if

$$
\begin{aligned}
A^{q-1} \geq(l-k)\left(\frac{k}{l}\right)^{1 \frac{q \lambda-\lambda}{\sigma \lambda}+1} & =\left(\frac{1}{\sigma}\left(\frac{A^{\lambda \sigma-1}}{a^{\lambda+1}}\right)\left(\frac{\sigma(\lambda+1)}{\sigma \lambda-1}\right)^{\lambda} N+\frac{1}{q-1}\right) \\
& \times\left(\frac{\frac{1}{\sigma}\left(\frac{A^{\lambda \sigma-1}}{a^{\lambda+1}}\right)\left(\frac{\sigma(\lambda+1)}{\sigma \lambda-1}\right)^{\lambda} \frac{\lambda+1}{\sigma \lambda-1}+\alpha \frac{\lambda+1}{\sigma \lambda-1}}{\frac{1}{\sigma}\left(\frac{A^{\lambda \sigma-1}}{a^{\lambda+1}}\right)\left(\frac{\sigma(\lambda+1)}{\sigma \lambda-1}\right)^{\lambda}\left(N+\frac{\lambda+1}{\sigma \lambda-1}\right)+\alpha \frac{\lambda+1}{\sigma \lambda-1}+\frac{1}{q-1}}\right)^{\frac{q \lambda-\lambda}{\sigma \lambda}+1}
\end{aligned}
$$

Clearly, For any fixed $\lambda, m, q$ and $N$, (3.7) is true if we choose $A$ large enough, and at the same vary $a$ such that the ratio $\left(A^{\lambda \sigma-1}\right) /\left(a^{\lambda+1}\right)$ keeps stable. Moreover, $A$ is increasing with respect to $N$. This completes the proof.

\section{References}

[1] Z. L. Liang, "Blow up Rate for a Porous Medium Equation with Power Nonlinearity,” Nonlinear Analysis: Theory, Methods \& Applications, Vol. 73, No. 11, 2010, pp. 3507-3512. doi:10.1016/j.na.2010.06.078

[2] A. Samarskii, V. Galaktionov and V. Kurdyumov, "Blow up in Quasilinear Parabolic Equations,” Walter de Gruyter, Berlin, 1995.

[3] A. Tersenov, "Space Dimension Can Prevent the Blowup of Solution for Parabolic Problems,” Electronic Journal of Differential Equations Vol. 165, No. 165, 2007, pp. 1-
6.

[4] J. N. Zhao, "Existence and Nonexistence of Solutions for $u_{t}=\operatorname{div}\left(|\nabla u|^{p-2} \nabla u\right)+f(\nabla u, u, x, t)$," Journal of Mathematical Analysis and Applications, Vol. 172, No. 1, 1993, pp. 130-146. doi:10.1006/jmaa.1993.1012

[5] D. Andreucci and A. F. Tedeev, "Universal Bounds at the Blow-Up Time for Nonlinear Parabolic Equation,” Advances in Differential Equations, Vol. 10, No. 1, 2005, pp. 89-120.

[6] Z. Q. Wu, J. N. Zhao and J. X. Yin, et al., "Nonlinear Diffusion Equations,” World Scientific Publishing Co., Inc., River Edge, 2001.

[7] Z. L. Liang and J. N. Zhao, "Localization for the Evolution p-Laplacian Equation with Strongly Nonlinear Source Term," Journal of Differential Equations, Vol. 246, No. 1, 2009, pp. 391-407. doi:10.1016/j.jde.2008.07.038 